\title{
Comparaison des mises en œuvre d'un même dispositif d'aide dans des contextes différents
}

Comparison of implementations of an assistance system in different contexts

Karine Millon-Faure, Laurent Theis, Teresa Assude, Jeanne Koudogbo, Jeannette Tambone et Marie-Pier Morin

\section{(2) OpenEdition}

\section{Journals}

Édition électronique

URL : https://journals.openedition.org/educationdidactique/3477

DOI : 10.4000/educationdidactique.3477

ISSN : 2111-4838

Éditeur

Presses universitaires de Rennes

\section{Édition imprimée}

Date de publication : 27 décembre 2018

Pagination : 43-64

ISBN : 978-2-7535-7774-9

ISSN : 1956-3485

Référence électronique

Karine Millon-Faure, Laurent Theis, Teresa Assude, Jeanne Koudogbo, Jeannette Tambone et MariePier Morin, «Comparaison des mises en œuvre d'un même dispositif d'aide dans des contextes différents », Éducation et didactique [En ligne], 12-3 | 2018, mis en ligne le 01 janvier 2021, consulté le 25 avril 2022. URL : http://journals.openedition.org/educationdidactique/3477 ; DOI : https://doi.org/ 10.4000/educationdidactique.3477 


\title{
COMPARAISON DES MISES EN GEUVRE D'UN MÊME DISPOSITIF D'AIDE DANS DES CONTEXTES DIFFÉRENTS
}

\author{
Karine Millon-Fauré \\ Équipe ADEF, Aix-Marseille Université, \\ Laurent Theis \\ Université de Sherbrooke, \\ Teresa Assude \\ Équipe ADEF, Aix-Marseille Université, \\ Jeanne Koudogbo \\ Université de Sherbrooke, \\ Jeannette Tambone \\ Équipe ADEF, Aix-Marseille Université, \\ Marie-Pier Morin \\ Université de Sherbrooke
}

Lors d'une recherche collaborative (Bednarz, 2013) menée avec des enseignantes d'une école primaire québécoise (Theis $\&$ al., 2014), nous avons travaillé à l'élaboration et la mise en œuvre de dispositifs d'aide destinés à préparer certains élèves en difficulté en mathématiques à la séance en classe entière qui devait suivre. En nous appuyant sur le triplet des genèses (Sensevy, Mercier \& Schubauer-Leoni, 2000), nous avons modélisé trois fonctions de ce dispositif d'aide : une fonction mésogénétique, une fonction chronogénétique et une fonction topogénétique. Notre question est à présent de savoir s'il serait possible d'amener d'autres enseignants à s'approprier ce dispositif tout en préservant ces fonctions. Pour cela, nous avons étudié sa mise en place par un enseignant, n'ayant pas participé à la conception de ce dispositif et nous l'avons comparée aux mises en œuvre observées lors de notre recherche collaborative.

Mots-clés : dispositif d'aide, mathématiques, mésogénèse, chronogénèse, topogénèse, recherche collaborative.

\section{Comparison of implementations of an assistance system in different contexts}

During a collaborative research (Bednarz, 2013) conducted with teachers from a Quebec elementary school (Theis \& al., 2014), we have been working on the development and implementation of support device to prepare some difficulty students in mathematics at the whole class session that was to follow. By relying on on the "triplet des genèses" (Sensevy, Mercier $\mathcal{E}$ Schubauer-Leoni, 2000), we modelled three functions of this aid device: a mesogenetic function, a chronogenetic function and a topogenetic function. In this way, our question is now whether it would be possible to encourage other teachers to take ownership of this system while by preserving these functions. To do this, we studied its implementation by a teacher, who did not participate to the design of this device and we compared it to the implementations observed during our research collaborative.

Keywords: support systems, mathematics, mesogenesis, chronogenesis, topogenesis, collaborative research. 
Karine Millon-Fauré \& al.

Lors d'une recherche menée au Québec (Theis $\varepsilon$ al., 2014), nous avons étudié l'élaboration et la mise en œuvre d'un dispositif particulier d'aide aux élèves en difficulté dans la résolution de problèmes en mathématiques. L'originalité de cette expérimentation réside dans la chronologie choisie : contrairement aux séances de remédiation qui visent un retour sur les savoirs rencontrés en classe entière, une fois les difficultés repérées, ces dispositifs d'aide se situent en amont du travail en classe entière. Notre recherche peut être qualifiée de collaborative (Bednarz, 2013) dans la mesure où il ne s'agit pas d'une recherche sur les enseignants mais avec les enseignants: les enseignantes impliquées dans le projet ont ainsi participé non seulement à l'élaboration des séances (l'idée initiale du dispositif étudié vient d'ailleurs d'une de ces enseignantes), mais également à leur analyse. Nous avons ainsi pu étudier quatre mises en œuvre différentes dans une même école primaire et nous avons peu à peu affiné notre description des fonctions de ce dispositif d'aide (Theis \& al., 2014; Assude \& al., 2016 a.).

La question que nous nous sommes alors posée portait sur les possibilités d'appropriation de notre dispositif par les professeurs, ce qui nous a amenés à proposer sa mise en œuvre à une enseignante française n'ayant pas participé à notre recherche collaborative. Deux paramètres, essentiellement, distinguaient cette expérimentation de celles menées précédemment : l'absence d'accompagnement de l'enseignante et la spécificité des contraintes institutionnelles en France par rapport au Québec. Nous avons alors observé comment cette enseignante s'appropriait ce dispositif et nous avons cherché à déterminer si les fonctions repérées dans les premières expérimentations se retrouvaient ou non dans cette nouvelle mise en œuvre. Afin de présenter nos résultats, nous allons tout d'abord exposer certains appuis théoriques ainsi qu'une description de la modélisation de ce dispositif élaborée lors de nos analyses précédentes. Dans une deuxième partie, nous présentons brièvement certains éléments méthodologiques permettant de mieux comprendre les conditions d'expérimentation. Enfin, nous cherchons à comparer cette dernière mise en œuvre aux précédentes afin de déterminer si nous observons les mêmes caractéristiques, d'une part du point de vue de la structure de ces dispositifs, et d'autre part en ce qui concerne leurs fonctions.

\section{APPUIS THÉORIQUES}

Contrairement à certaines recherches, généralement anglo-saxonnes, centrées sur l'élève en difficulté et notamment sur ses processus cognitifs, nous privilégions une approche systémique qui nous amène à considérer simultanément les élèves, l'enseignant, l'enjeu de savoir ainsi que leurs interactions. À la suite d'autres recherches (Giroux, 2014; Mary $\mathcal{E}$ al., 2014), nous considérons cet ensemble comme une unité insécable et nous pensons que les difficultés rencontrées par l'élève ne peuvent se comprendre qu'en tant que difficultés du système didactique dans sa globalité.

Reprenant la classification de Chevallard (1995), nous qualifions alors la classe de système didactique principal et nous nous intéressons aux systèmes didactiques auxiliaires composés d'une partie des actants du système principal : «Un système dit principal engendre (et, dans une certaine mesure, commande) un ensemble de systèmes didactiques dits auxiliaires qui, comme leur nom le dit assez, viennent en aide au système didactique principal » (Chevallard, 2010, p. 10). Les systèmes didactiques auxiliaires (que nous notons dans cet article SDA) entretiennent donc une relation de dépendance avec leur système didactique principal (désigné sous l'acronyme SDP), notamment du point de vue des enjeux de savoir. Les SDA habituels (Leutenegger, 2009; Toullec-Théry \& Marlot, 2012; Tambone, 2014) correspondent à des dispositifs d'aide permettant à l'enseignant de reprendre avec une partie de ses élèves certains points de la leçon sur lesquels ces derniers ont rencontré des difficultés dans le système didactique principal. Nos recherches portent sur un type de SDA différent, puisque ces dispositifs d'aide interviennent en amont des séances en classe entière et visent à préparer certains élèves à l'activité qui va se dérouler dans le SDP. Ce procédé peut être éventuellement complété par un SDA post, indiqué en italique dans le schéma ci-dessous car nous n'en parlerons pas dans cet article.

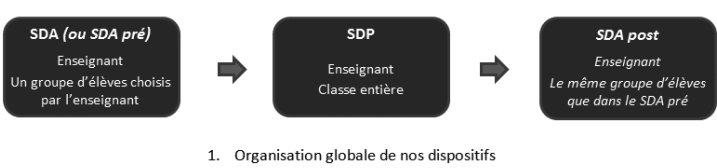

Pour étudier ces dispositifs, nous nous appuyons tout d'abord sur la théorie anthropologique du didac- 
tique (Chevallard, 1999) et plus spécifiquement sur l'étude des praxéologies mais aussi sur le triplet des genèses (Sensevy, Mercier \& Schubauer-Leoni, 2000). Les praxéologies permettent de décrire les objets de savoirs comme étant constitués de deux blocs: le bloc praxis composé des types de tâche et des techniques et le bloc logos correspondant au discours sur la technique (la technologie et la théorie). Enseigner un objet de savoir consiste donc à organiser l'étude de chacune des composantes de la praxéologie associée. Nous utilisons également dans nos analyses le triplet des genèses (mésogenèse, topogenèse, chronogenèse) que Sensevy, Mercier \& Schubauer-Leoni (Ibid.) définissent ainsi :

« au sein du système didactique, le professeur doit agir (définir, réguler, dévoluer, instituer) pour :

- produire les lieux du professeur et de l'élève (effet de topogenèse);

- produire les temps de l'enseignement et de l'apprentissage (effet de chronogenèse) ;

- produire les objets des milieux des situations et l'organisation des rapports à ces objets (effet de mésogenèse) »(Sensevy et al., Ibid., p. 267).

Ces concepts nous ont permis de mettre en exergue, dans nos SDA, trois fonctions fortement liées (Theis E al., 2014; Theis \& al., 2016; Assude \& al., 2016 a.; Assude \& al., 2016 b.).

Nous avons tout d'abord montré que les SDA que nous avons étudiés présentaient une fonction mésogénétique. En effet, pendant ces dispositifs d'aide, vont être travaillés certains des objets qui figurent dans le milieu de la situation prévue pour le SDP. Ainsi les élèves sont amenés à manipuler certains concepts, mathématiques ou non, et à rencontrer certains éléments de praxéologies (types de tâches ou techniques) qui se révéleront utiles lors du retour dans la classe.

Nous avons également mis en exergue une fonction chronogénétique. Dans les dispositifs que nous avons observés au cours de notre recherche collaborative, la séance de SDA était située en amont du SDP, ce qui permettait aux élèves présents de savoir avant leurs camarades de quoi il allait s'agir (« savoir plus avant ", Assude \& al., 2016 b., p. 211). Pour autant, il convient de souligner que ces SDA ne constituent pas une simple répétition de ce qui va se jouer en classe : les tâches proposées et le rythme (Assude, 2005) ne sont pas les mêmes. Ainsi, certains concepts, concernant par exemple les consignes ou certaines contraintes de la situation, qui sont à peine abordés dans le SDP, ont fait l'objet de discussions entre l'enseignante et les élèves dans le SDA.

Par ailleurs, tout comme cela a déjà pu être montré dans des SDA " habituels », c'est-à-dire postérieurs à la séance (Leutenegger, 2009; ToullecThéry \& Marlot, 2012; Tambone, 2014), nous avons pu constater que le temps didactique n'avançait pas durant ces dispositifs d'aide. Nous avons toutefois établi qu'il y avait bien une temporalité qui avançait durant nos SDA (Assude $\mathcal{E}$ al., 2016 b.) puisque ces derniers engageaient les élèves dans une meilleure connaissance de certaines techniques constituant les praxéologies qui allaient être mises en jeu dans le SDP. Nous avons nommé cette temporalité " temps praxéologique » et nous l'avons définie comme la temporalité qui rend compte de l'évolution de chacune des composantes d'une praxéologie (connaissance d'un type de tâches, d'une technique voire d'un élément de technologie...). Ainsi une avancée du temps didactique s'accompagne nécessairement d'une avancée du temps praxéologique, la réciproque n'étant pas vraie : un travail sur des praxéologies concernant des objets « désensibilisés » ou des objets « insensibles ${ }^{1}$ » du milieu (Mercier, 1996; Millon-Fauré, 2011), c'est-à-dire non pris en compte officiellement par l'institution, peut constituer une avancée du temps praxéologique, mais pas du temps didactique.

Enfin, la dernière fonction dont nous parlons dans cet article est la fonction topogénétique. Nous avons en effet pu observer que les élèves du SDA, décrits par leurs enseignantes comme s'investissant peu dans les activités de la classe, parvenaient à prendre position dans le topos d'élève (Schubauer-Leoni, Leutenegger, Ligozat \& Fluckiger, 2007; Tambone, 2014): réponses aux questions de l'enseignante, interventions spontanées... Nous avons également remarqué que, dans la séance en classe entière, ces mêmes élèves adoptaient une attitude comparable à celle de leurs camarades (réalisation des tâches demandées, investissement dans le travail de groupe, participation aux discussions en classe entière...). Sans pouvoir affirmer qu'il s'agit là d'une des conséquences du SDA, l'aspect inhabituel de leur comportement nous amène à conjecturer que la mise en place de ces dispositifs en amont de la séance pourrait permettre à certains élèves en difficulté de retrouver au moins temporairement un véritable topos d'élève. 
Karine Millon-Fauré \& al.

Ces fonctions de nos dispositifs ont pu être observées dans le cadre d'une recherche collaborative qui réunissait huit enseignantes d'une même école primaire québécoise et six chercheurs autour d'une préoccupation commune : l'aide aux élèves en difficulté lors de la résolution de problèmes. Notre question est de savoir si ce dispositif présenterait les mêmes fonctions en dehors d'une recherche collaborative afin de déterminer s'il serait alors possible de le diffuser auprès d'autres enseignants. En effet, même si la conception de ce dispositif a bénéficié de l'expertise de praticiens, ce qui augmente ses chances de viabilité dans la classe, cela ne garantit pas pour autant sa reproductibilité par un enseignant n'ayant pas participé à la recherche collaborative. Le cadre induit par ce type de recherches influence les conditions de mise en œuvre sur deux points tout au moins. Tout d'abord l'organisation des séances de classe est discutée par les enseignants et les chercheurs conjointement, chacun utilisant son expérience spécifique (issue de la pratique ou de la recherche) pour enrichir la réflexion commune concernant le choix des tâches ou le déroulement de l'activité :

« La recherche collaborative, en ce sens, s'articule autour de projets dont l'intérêt d'investigation repose sur la compréhension que les praticiens, en interaction avec le chercheur, vont construire autour de l'exploration, en contexte réel, d'un aspect qui concerne leur pratique professionnelle.» (Desgagné, 1997, p. 373).

Par ailleurs, une recherche collaborative peut également contribuer à la formation non seulement des chercheurs mais également des enseignants qui y travaillent :

« la participation au projet de recherche et au questionnement qu'il soulève va permettre aux praticiens, soutenus par le chercheur, d'éclairer, voire d'élargir leur vision de la pratique »(Desgagné \& Bednarz, 2005, p. 249).

Ce développement professionnel, s'il est atteint, est susceptible d'influer sur les pratiques des enseignants, notamment lors du déroulement des séances. Dans ces conditions, il est légitime de se demander comment un autre enseignant peut s'approprier ce dispositif : les séances qu'il concevra et mettra en œuvre présenteront-elles les mêmes fonctions que celles observées dans notre recherche collaborative?

\section{ÉLÉMENTS MÉTHODOLOGIQUES}

Dans le cadre de notre recherche collaborative québécoise, nous avons pu étudier quatre expérimentations et parmi celles-ci, deux portaient sur la description de figures géométriques : une expérimentation dans la classe de Sylvie avec des élèves de 8-9 ans et une dans celle de Sophie avec des élèves de 7-8 ans (Theis \& al., 2016). Afin de faciliter la comparaison, nous voulions observer la mise en œuvre par un enseignant extérieur à notre recherche d'un dispositif centré sur le même type de problème mathématique. Notons que, comme cette nouvelle mise en œuvre s'est déroulée dans une école française, il nous faudra prendre en compte l'influence éventuelle des contraintes institutionnelles spécifiques à chaque pays sur les fonctions du dispositif d'aide.

En novembre 2015, il a donc été proposé à une enseignante française, Cléa, exerçant auprès d'une classe de CM2 (élèves de 10 ans) dans une école ordinaire de Marseille, de mettre en place une situation de communication sur la description de figures géométriques dans sa classe (un binôme d'élèves devait décrire une figure géométrique pour qu'un autre puisse la reproduire). Nous avions demandé à l'enseignante de faire précéder cette séance, d'un temps de travail avec un groupe d'élèves en difficulté afin de faciliter leur implication dans le SDP. Mis à part ces indications, nous l'avions volontairement laissée libre de choisir les tâches qu'elle allait proposer, afin de pouvoir étudier la façon dont elle allait s'approprier ces directives et comparer cette mise en œuvre aux précédentes.

Pour la séance de SDA, l'enseignante a choisi les six élèves (Fabio, Ode, Lucas, Léa, Arthur et Henry) qui, selon elle, étaient susceptibles de rencontrer le plus de difficulté lors du problème prévu dans le SDP. Il leur était demandé d'écrire, à partir des vignettes ci-dessous, les instructions correspondant à la construction de la figure finale (voir le tableau synoptique en annexe 2$)^{2}$ : 
Karine Millon-Fauré \& al.

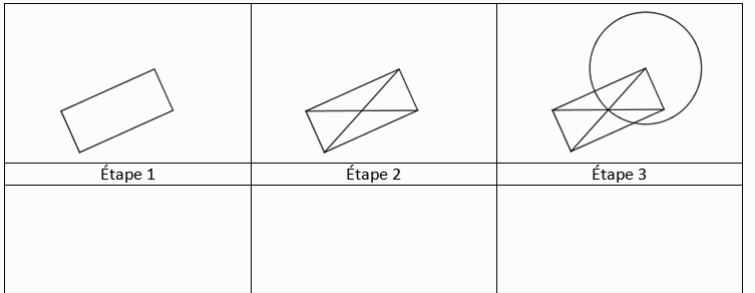

Cette séance avec un effectif réduit d'élèves a été suivie de deux séances en classe entière qui constituent le système didactique principal. Durant la première séance du SDP, les élèves répartis en binômes ont reçu une des cinq figures géométriques ci-dessous qu'ils devaient décrire afin que leurs camarades puissent effectuer la construction sans disposer du dessin initial (voir le tableau synoptique en annexe 3) :
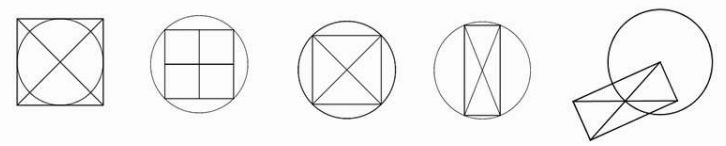

Précisons que la dernière figure, identique à celle du SDA, n'a pas été donnée aux élèves ayant bénéficié du dispositif d'aide. Durant la seconde séance, chaque binôme a reçu les instructions produites précédemment par une autre équipe d'élèves et a tenté de construire la figure correspondante. Enfin les quatre élèves qui avaient échangé leurs descriptions se sont regroupés pour comparer les tracés obtenus avec les figures originales et réfléchir aux causes des écarts observés (voir le tableau synoptique en annexe 4). Même si des séances ultérieures et des évaluations d'élèves sont venues compléter ce dispositif, nous n'étudions dans cet article que les trois séances précédemment décrites.

Deux dispositifs d'enregistrement vidéo et un dispositif d'enregistrement audio ont été utilisés pendant chacune de ces séances ce qui nous a permis de suivre simultanément le travail de trois groupes d'élèves. Par ailleurs, les productions des élèves ont systématiquement été relevées et, entre les séances de classe, des entretiens avec l'enseignante ont été organisés. Nous avons confronté ces données aux analyses que nous avions précédemment faites concernant les SDA québécois (expérimentations qui avaient été réalisées dans des conditions méthodologiques similaires) et nous avons cherché à déterminer quelles étaient les similitudes, mais également les spécificités de cette nouvelle mise en œuvre par rapport aux précédentes.

\section{COMPARAISON DE LA STRUCTURE DES SDA}

\section{Des différences dans l'organisation matérielle}

Si l'on regarde tout d'abord du point de vue de l'organisation matérielle, on constate que le temps consacré au système didactique auxiliaire est approximativement deux fois plus court au Québec qu'en France (une vingtaine de minutes et une quarantaine de minutes respectivement). En effet, en France, l'enseignante a utilisé un dispositif préexistant et nommé « Activités Pédagogiques Complémentaires » ou APC. Il s'agit de trente-six heures annuelles consacrées à l'accompagnement d'un groupe restreint d'élèves sur un temps extérieur aux vingt-quatre heures hebdomadaires d'enseignement obligatoire ${ }^{3}$. Ce temps consacré aux activités pédagogiques complémentaires est généralement réparti dans la semaine en plusieurs séances, (par exemple en deux séances de trente minutes, comme c'est le cas dans l'école observée) et les élèves choisis par l'enseignante (avec l'accord des parents) pour participer à ce dispositif varient au cours de l'année.

Au Québec par contre, aucun dispositif institutionnel ne permet de travailler avec seulement une partie de la classe. L'organisation des SDA a donc été plus délicate. Ainsi une enseignante a profité d'un moment où un étudiant stagiaire devait assurer un cours dans sa classe pour isoler certains élèves. Par ailleurs, les enseignantes québécoises ont choisi une salle différente de la salle de cours habituelle: les tables toutes face à face, semblent organisées pour une réunion afin de faciliter les interactions. D'ailleurs, l'essentiel de la séance est consacré à une grande discussion entre tous les membres du groupe (élèves et enseignante). En France, au contraire, le fait d'utiliser la salle de classe, où tous les pupitres sont tournés vers le tableau, et le fait que la séance soit organisée principalement autour d'un travail individuel puis en binôme, quasiment sans interventions de l'enseignante, confèrent à ce système didactique auxiliaire un format assez proche des séances classiques. 
Karine Millon-Fauré \& al.

\section{Une absence de justification du dispositif}

La possibilité d'inscrire le système didactique auxiliaire dans un dispositif institutionnel induit une autre différence majeure entre la mise en œuvre dans la classe française et celles observées au Québec. Les écoliers français (ainsi que leurs parents) connaissent l'existence de ces activités complémentaires dans leur établissement et savent qu'ils seront éventuellement choisis à un moment ou à un autre pour y participer. Cinq des six élèves de notre système didactique auxiliaire ont déjà été retenus pour des activités complémentaires depuis la rentrée et il semble donc inutile à l'enseignante de justifier leur participation à cette séance.

Au Québec, la situation est tout autre : ce type d'expérimentation n'ayant jamais été organisé dans leur classe, les enseignantes se doivent de légitimer d'une part la mise en place de ce dispositif et d'autre part le choix des élèves qui y participent. C'est pourquoi tous les systèmes didactiques auxiliaires québécois que nous avons observés débutent par une explication rapide de l'intérêt du dispositif. Selon les enseignantes, les arguments avancés sont les suivants : il offre la possibilité de découvrir la situation avant le reste de la classe; il permet aux élèves présents de travailler en petit effectif; il constitue un « entraînement » pour les travaux en classe entière. Les enseignantes sont également amenées à expliciter les critères qui les ont conduit à choisir ces élèves-là. Ainsi Sylvie précise qu'ils ont été choisis en raison de leurs difficultés habituelles pour s'investir dans les activités mathématiques. Tout ceci contribue à souligner ce que ce dispositif peut apporter à ces élèves, une fois qu'ils retourneront en classe. Il est possible que ce type d'explications ait été donné par l'enseignante française lorsqu'elle a présenté les activités pédagogiques complémentaires en début d'année. Toutefois, une fois le dispositif installé, ces justifications n'apparaissent plus.

\section{Des attentes institutionnelles différentes}

Si l'on se restreint aux trois dispositifs qui portaient sur la situation de description de figures en géométrie (deux dispositifs québécois et un français), on observe des différences, notamment en ce qui concerne les objectifs d'enseignement. Les deux enseignantes québécoises cherchaient à travailler, au travers de cette situation, sur les propriétés de certains parallélogrammes. Les figures proposées étaient donc élémentaires (voir annexe 1), mais le fait de ne pouvoir les nommer contraignait les élèves à utiliser les propriétés de leurs angles ou de leurs côtés pour les caractériser. L'enseignante française, quant à elle, a vu dans cette situation une possibilité de travailler sur les programmes de construction (c'est d'ailleurs, l'objectif qu'elle stipule dans sa fiche de préparation). Elle a par conséquent choisi des figures complexes, composées de cercles et de parallélogrammes. De plus, alors que les enseignantes québécoises avaient demandé aux élèves de décrire la figure, Cléa oriente dès la première consigne vers les programmes de construction, même si cette expression n'est pas prononcée :

«Vous allez écrire, rédiger des instructions qui vont amener quelqu'un qui n'a pas vu ce que vous avez, votre figure, qui n'a jamais vu votre figure et qui va essayer d'après vos instructions de refaire la même figure. »

Il ne s'agit pas ici de donner certains éléments caractéristiques de la figure finale, mais d'expliciter les instructions, correspondant aux différentes étapes à suivre pour construire cette figure. On retrouve là les deux grandes catégories mises en exergue par Lahanier-Reuter (1999) lorsqu'elle analyse les difficultés rencontrées par les élèves lors des activités de description de figures géométriques : les descriptions correspondant à ce qui est vu et les descriptions correspondant à la construction de la figure. Les enseignantes françaises et québécoises interprètent donc de manière différente le type de tâches « décrire une figure » et ceci peut s'expliquer par le contenu des programmes propres à chaque pays. En France, à côté des tâches de description (jeu du portrait...) figure explicitement dans les programmes de l'école primaire, l'expression " programmes de construction $»$ :

« Tracer une figure simple à partir d'un programme de construction ou en suivant des consignes [...] Tracer une figure (sur papier uni, quadrillé ou pointé), à partir d'un programme de construction ou d'un dessin à main levée $e^{4}$ »

Les documents d'accompagnement du cycle $3^{5}$ conseillent de travailler sur " l'élaboration d'un message contenant toutes les informations néces- 
Karine Millon-Fauré \& al.

saires à la reproduction de la figure » et notamment "l'énoncé de la suite des étapes qui permettent de construire la figure (programme de construction) ${ }^{6} »$. Les programmes du collège soulignent également l'intérêt de ce type d'activité qui légitime certaines exigences de précision, surtout en ce qui concerne le recours au lexique mathématique. En outre, même si les tâches de description sont toujours présentes, la place des programmes de construction dans l'enseignement au nouveau cycle $3^{7}$ s'accentue encore dans les programmes de la rentrée 2016 : d'une part, l'élaboration d'un programme de construction fait à présent partie des attendus ( « réaliser, compléter et rédiger un programme de construction $\left.»^{8}\right)$, d'autre part, le programme de construction est présenté comme un moyen de préparer les élèves à l'algorithmique (« [Les activités spatiales et géométriques] constituent des moments privilégiés pour une première initiation à la programmation, notamment à travers la programmation de déplacements ou de construction de figures $»^{9}$ ).

Dans le programme québécois, au contraire, l'expression « programme de construction» n'est pas mentionnée. On trouve par contre des allusions à des descriptions de figures géométriques, mais dans un contexte légèrement différent que celui dans lequel cette expression est habituellement utilisée en France. Il s'agit dans le programme québécois, de description de figures « élémentaires » que les élèves connaissent et savent nommer ( Description du carré, du rectangle, du triangle et du losange [...] Description des quadrilatères dont le trapèze et le parallélogramme [etc.] ${ }^{10}$ ). Les élèves doivent donc être en mesure d'identifier la figure proposée, puis de donner ses propriétés caractéristiques. Ces considérations sur les programmes respectifs de chaque pays éclairent les choix des enseignantes en ce qui concerne, d'une part, les figures à décrire et, d'autre part, les consignes données aux élèves.

\section{Des types de tâches différents}

Enfin, une dernière différence dans la structure apparaît entre les deux dispositifs québécois portant sur les descriptions de figures et le dispositif français. L'activité prévue dans les SDP québécois est constituée de quatre types de tâches emboîtés (autrement dit la réalisation de chaque type de tâches s'avère indispensable au suivant) :
- T1 - décrire une figure

- T2 - tracer une figure à partir d'une description

- T3 - comparer les deux figures

- T4 - déterminer les causes des différences entre la figure originale et sa reproduction

Dans le SDA, les enseignantes québécoises choisissent toutes deux de travailler sur le premier de ces quatre types de tâche : les élèves doivent décrire, sans les nommer, un carré ou un triangle (dont les figures sont présentées sans codage et sans que les points ne soient nommés $\left.{ }^{11}\right)$. Nous pouvons relier ce choix à leur préoccupation récurrente de faciliter l'entrée de ces élèves dans l'activité, d'autant plus qu'ici un échec dans le premier type de tâches rendrait le reste de l'activité irréalisable.

Dans le SDP mis en place en France, si les derniers types de tâche (T2, T3 et T4) sont identiques à ceux observés au Québec, le premier type de tâche diffère quelque peu puisque, l'enseignante attend des élèves la production d'un programme de construction. Ce type de tâche peut se découper en quatre sous-types de tâche : T1.1 - décomposer une figure complexe en figures élémentaires; T1.2 - repérer la position relative des figures élémentaires les unes par rapport aux autres; T1.3 - identifier une chronologie possible pour la construction; T1.4 - formuler des instructions. Contrairement à ses collègues québécoises qui cherchaient à faciliter l'entrée dans l'activité, l'enseignante française ne va pas proposer à ses élèves du SDA de travailler sur les premiers soustypes de tâches qu'ils rencontreront dans le SDP. Elle décide de prendre à sa charge les sous-types de tâches T1.1 et T1.3 (le type de tâche T1.2 est resté implicite) et de leur donner le " film» de la construction, c'est-à-dire les vignettes caractérisant à chaque fois l'ajout d'une figure élémentaire à la figure précédente, l'ordre de ces vignettes indiquant l'ordre dans lequel a été exécuté le tracé. En effet, l'enseignante voulait dans le SDA soulager les élèves des autres types de tâches afin qu'ils puissent se centrer sur le type de tâches qui lui paraît le plus problématique, à savoir la formulation des instructions :

$\mathrm{P}$ : [j'attends] des termes mathématiques précis [...] ils n'ont pas encore le problème qui va se poser de l'ordre puisque je leur donne l'ordre, ils ont l'ordre des figures, donc il va falloir s'atteler à vraiment utiliser un vocabulaire précis, que ce soit les verbes, c'est-à-dire nommer, tracer, placer, rien que ces 3 verbes, bien les 
Karine Millon-Fauré \& al.

employer et un rectangle qu'on va tracer et surtout le nommer.

Il faut noter que Cléa est moins préoccupée que les enseignantes québécoises par le fait de faciliter l'entrée dans la tâche. Les critères qui ont guidé son choix des élèves concernés par le SDA portent davantage sur les problèmes d'expression et notamment de maîtrise du lexique mathématique, que sur des difficultés à s'investir dans une activité.

\section{FONCTION MÉSOGÉNÉTIQUE}

\section{Une absence de présentation de la situation du SDP}

Nous avions observé que dans les SDA québécois, les enseignantes avaient systématiquement décrit la situation qui allait être présentée en classe entière, parfois même avec plus de détails que lors des explications du SDP.

A contrario, durant le SDA français, l'enseignante ne fait aucune allusion à l'activité prévue en classe entière. Toutefois la tâche proposée dans le SDA français conduit les élèves à une production proche de celle attendue dans le système didactique principal et certains élèves vont effectivement établir un lien entre ces deux activités. Ainsi un des élèves du SDA s'exclame en découvrant la situation proposée à la classe « c'est ce qu'on a fait en APC ». En outre, certains des objets introduits durant le dispositif d'aide - les concepts de rectangle, diagonale, cercle - s'avèrent également utiles pour appréhender le milieu initial de la situation du SDP, puisque ce sont ces figures qui composent les figures complexes que les élèves auront à décrire en classe entière.

\section{D'un espace de questionnement ouvert à un espace de questions très dirigées}

Pour travailler dans le SDA sur ces objets utiles au SDP, les enseignantes québécoises ont mis en place une organisation particulière que nous avons appelée "espace de questionnement». Chacune, par ses questions, pousse les élèves à expliciter leurs connaissances et à affiner leurs techniques :
P : Alors, c'est spécial, quand même, hein? Parce que tout le monde a la même. Le même dessin. Mais, vous avez décrit de façons différentes. Selon vous, quelle description permettrait... Quelle description permettrait à un... à un copain, à une autre personne, de dessiner le plus pareil possible, le plus identique possible, cette... ce carré-là. [...]

E : La mienne.

P : La tienne. Ta description. Pourquoi?

L'objectif de cet espace de questionnement n'est pas tant de déboucher sur des réponses correctes (les enseignantes ne rectifient pas les erreurs des élèves et évitent d'introduire elles-mêmes les objets de savoirs dont ils auraient besoin), que d'amener les élèves à se poser les « bonnes » questions, questions auxquelles ils répondront éventuellement en classe entière. Le lien fort qui a été fait dès le départ avec le SDP permet aux enseignantes de ne pas finaliser le SDA : il est tout à fait possible de laisser les questions en suspens, puisque l'activité doit se poursuivre.

Dans le SDA français, par contre, l'organisation du travail ressemble beaucoup plus à celle d'une séance de mathématiques traditionnelle. Après un travail individuel, les élèves comparent leur production en binôme, puis vient un temps de mise en commun qui ressemble à une correction, notée au tableau, de l'exercice proposé. L'enseignante française pose des questions mais elles sont beaucoup plus ciblées, notamment en ce qui concerne le vocabulaire, que dans les SDA québécois :

$\mathrm{P}$ : Trace un cercle de centre $\mathrm{O}$ et de rayon... c'est quoi son rayon alors? Comment il s'appelle son rayon?

$\mathrm{E}: \mathrm{O}$ ?

$\mathrm{P}$ : O c'est son point d'intersection. C'est une distance. Il me faut une distance.

$\mathrm{E}: \mathrm{A}$ et $\mathrm{B}$ ?

$\mathrm{P}$ : A et B, non ce n'est pas ça le rayon. C'est quoi ça? Comment j'appelle ce segment? Comment j'appelle ce segment, Lucas? Tiens, ce segment qui part de là à là ? Je l'entends mais... et de rayon quoi? C'est quoi cette distance-là? [Elle pointe au tableau le rayon du cercle.]

Comment je peux l'appeler?

$$
\begin{aligned}
& \mathrm{E}: \mathrm{AO} \text {. } \\
& \mathrm{P}: \mathrm{AO} \text {, oui. Et de rayon... AO. }
\end{aligned}
$$

L'enseignante guide les réponses des élèves (« il me faut une distance »), et même les évalue ( «non »), ce qui n’apparaissait pas dans les dispositifs québé- 
cois. Elle tient à obtenir dans le SDA une production exacte qui pourra leur servir d'exemple pour l'activité prévue en classe entière. La séance se termine d'ailleurs par la copie de cette correction sur les feuillesélèves. Ceci peut s'expliquer par le fait que Cléa n'a pas présenté ce temps de travail comme une préparation à la séance qui va se jouer en classe entière. Elle se sent donc obligée de finaliser l'activité à la fin du SDA alors que dans les dispositifs québécois, il s'agissait d'une ouverture pour le SDP : certaines questions peuvent donc rester sans réponse dans la mesure où elles seront reprises à la séance suivante.

\section{Une absence d'objets sensibles dans le SDA...}

Lors de notre analyse des dispositifs québécois, nous avons noté que le choix des objets sur lesquels les enseignantes ont choisi de travailler dans le SDA n'est pas anodin : il s'agit uniquement d'objets désensibilisés ou insensibles, le travail sur les objets sensibles étant réservé au SDP. Les enseignantes considèrent en effet le SDA comme un espace où l'on peut travailler sur des objets avec lesquels un rapport d'idonéité ${ }^{12}$ s'avère utile pour appréhender la situation du SDP, et qui ne nécessitent pas d'être approfondis en classe entière car ils ne constituent de réels obstacles que pour les élèves en difficulté.

De même, dans le SDA français, de nombreux objets viennent compléter le milieu, mais qui sont tous des objets désensibilisés (angles droits, segment, côtés...). L'enseignante cherche à réactiver la mémoire didactique (Brousseau \& Centeno, 1991), notamment en ce qui concerne la manière de nommer une figure ou l'utilisation de termes spécifiques du langage mathématique :

$\mathrm{P}$ (en réponse à un élève qui avait nommé $\mathrm{A}, \mathrm{B}, \mathrm{C}, \mathrm{D}$ le rectangle $\mathrm{ABCD})$ : « Attention, quand on appelle un rectangle, on nomme les 4 sommets sans virgule, tu te rappelles?»[...]

$\mathrm{P}:$ « Est-ce que vous rappelez comment on appelle ces deux droites qui partent d'un sommet d'une figure pour aller au sommet opposé dans un quadrilatère? »

... et l'apparition d'objets migrants (Tambone \& Mercier, 2003; Tambone, 2014)

Sur le plan mésogénétique, les SDA québécois ont également provoqué l'apparition dans le système didactique principal, de certains objets qui avaient été travaillés dans le système didactique auxiliaire, que ce soit des techniques ou des concepts. Ainsi, au début des deux SDP concernant la description de figures, des informations en provenance du SDA vont permettre aux élèves de mieux comprendre la tâche attendue : Sylvie fait référence à une technique utilisée dans le SDA et une des élèves de la classe de Sophie explique à ses camarades qu'il est interdit de donner l'initiale de la figure, comme cela avait été précisé en petit groupe. Ces objets du SDA qui sont venus s'ajouter dans le milieu du SDP sont des objets « migrants » (Tambone \& Mercier, 2003; Tambone, 2014), c'est-à-dire des objets qui transitent entre deux institutions et qui permettent aux enseignants et aux élèves d'établir des liens entre deux systèmes didactiques distincts.

Dans le SDP français, on note également l'apparition d'objets en provenance du SDA. Ainsi, plusieurs élèves du SDA pensent à utiliser le mot « diagonale » alors que d'autres élèves, a priori moins en difficulté, ont utilisé des périphrases : " prends les sommets haut gauche et trace un segment puis fais pareil avec le sommet haut droit et le sommet bas gauche »; « on fait une croix, au milieu »; « deux segments qui partent des angles droits ». De plus, dès le début de la séance, Ode, propose d'ajouter sur la figure des codages, objets évoqués dans le SDA. Enfin, seuls deux binômes composés d'élèves du SDA pensent à nommer les points de la figure et à utiliser ces informations pour leur programme de construction, comme cela avait été proposé dans le SDA :

Fabio (qui lit son programme de construction) : «On prend des outils de géométrie et une feuille que le maître ou la maîtresse donne. On trace un cercle et dans le cercle on dessine un carré on prend notre règle et on part du sommet A jusqu'au sommet $C$. Après on reprend notre règle on part du sommet B au sommet $\mathrm{D}$ ».

Ode : Ça sert à rien de le dire car ils le savent. Une diagonale c'est toujours comme ça [elle joint du doigt 2 sommets opposés]. C'est jamais comme ça [elle montre un des côtés horizontaux]. Alors déjà ça fait une phrase en moins. (Épisode 2 dans l'annexe 3)

Nous constatons dans la description de Fabio que, cet élève pense à nommer les points, ce qu'il n'avait pas pensé à faire lors de sa première production du SDA. Par ailleurs, la remarque d'Ode montre qu'elle a mémorisé non seulement la recherche de concision 
Karine Millon-Fauré \& al.

d'un programme de construction qui n'a pas encore été évoquée dans le SDP et qu'elle s'est souvenue du terme « diagonale », introduit dans le SDA. On remarque toutefois dans la description de Fabio, que ce dernier mentionne encore les outils de géométrie alors qu'il avait été souligné dans le SDA que ce type d'information était inutile.

\section{FONCTION CHRONOGÉNÉTIQUE}

Le temps didactique n'avance pas dans nos SDA...

Lors de l'étude de la fonction chronogénétique des dispositifs québécois, nous avions montré que le temps didactique n'avançait pas durant les SDA (Assude $\mathcal{E}$ al., 2016 b.). Nous avions observé que si le processus de dévolution y était entamé, il n'y avait pas de processus d'institutionnalisation qui puisse attester d'une véritable avancée du temps didactique. En effet, le travail effectué dans le SDA portait essentiellement sur des objets désensibilisés ou insensibles nécessaires à l'engagement des élèves dans l'activité, la manipulation des objets sensibles, véritable enjeu de la situation, étant réservée au SDP. En outre, les élèves ne disposaient pas dans le SDA des moyens nécessaires pour contrôler leurs propos, que ce soit grâce aux rétroactions du milieu ${ }^{13}$ (absentes du SDA) ou aux réactions de l'enseignante. Ainsi, la construction des figures à partir des descriptions proposées, dispositif qui aurait pu permettre aux élèves d'éprouver la pertinence de leurs productions, n'aura lieu que dans le SDP. Nous avons également pu relever divers moments où l'enseignante refusait de donner aux élèves certains savoirs mathématiques dont ils avaient besoin :

$\mathrm{P}$ : Je veux pas vous dire ces mots-là à l'avance parce que là, demain, vous allez vraiment en savoir plus que les autres.

Dans le SDA français, il n'y a pas non plus de rétroactions du milieu. Les élèves ne disposent d'aucun élément de contrôle de leurs propositions. Toutefois, l'enseignante française guide bien davantage le travail des élèves que les enseignantes québécoises. Ainsi Cléa explicite dans le SDA quatre des sept points qui constituent l'institutionnalisation du SDP (les codages, le fait de nommer certains points de la figure, le fait d'utiliser des termes du lexique mathématique, l'inutilité de préciser les outils géométriques utilisés $)^{14}$. Pour autant, cela ne nous paraît pas correspondre à une avancée du temps didactique. En effet, il s'agit là de conventions correspondant à la forme canonique d'un programme de construction dont les motivations ne sont pas clairement explicitées durant le SDA. Ainsi, le recours à des termes du lexique mathématique semble uniquement relever des attentes de l'enseignante ( $\mathrm{P}$ : « soyons précis, en mathématiques, en géométrie, je veux des termes précis ») et l'utilisation des codages a été abordée sans que l'intérêt de cette pratique n'ait été discuté. De même, l'enseignante donne aux élèves deux contraintes qui peuvent apparaître contradictoires : il leur est en effet demandé d'une part d'être le plus précis possible, d'autre part de ne pas mentionner les instruments de géométrie nécessaires.

\section{... contrairement au temps praxéologique}

Nous avions observé que si, dans les SDA québécois, le temps didactique n'avançait pas, le temps praxéologique, lui, progressait bel et bien. En effet, tous les SDA québécois amènent les élèves à mettre en œuvre ou à expliciter (notamment grâce à l'espace de questionnement) des techniques qui pourront leur être utiles dans le SDP. Dans les deux SDA sur la description de figures, tous les élèves lisent leurs productions et les enseignantes insistent sur les techniques utilisées, ce qui peut faciliter leur diffusion :

E : tu as 3 sommets. Tu as 3 lignes. Ça ressemble à une pyramide. Ce n'est pas un carré ni..

$\mathrm{P}$ (Sylvie) : tu as dit ce que ce n'était pas. [...]

$\mathrm{E}: 3$ sommets très hauts, même pas gros, en forme de glissade très haute. Une moitié de volcan et une pyramide.

$\mathrm{P}:$ tu as donné des exemples. [...]

E : qu'est-ce qui ressemble à une pyramide à base triangulaire et un losange un peu.

$\mathrm{P}$ : c'est plus du langage mathématique, toi

Dans le SDA français, nous avons vu que les élèves avaient travaillé sur l'un des sous-types de tâches du SDP et l'enseignante les pousse également à formuler les techniques mises en jeu dans la rédaction des instructions d'un programme de construction. Ainsi, lorsque pendant la mise en commun, un 
Karine Millon-Fauré \& al.

élève propose pour la rédaction du programme de construction, l'instruction « Trace un cercle avec un compas », l'enseignante les amène à réfléchir à l'utilité de mentionner les instruments de géométrie utilisés :

$\mathrm{P}$ : Quand vous tracez un cercle, est-ce qu'on a besoin de mettre « à l'aide du compas»?

E : Non. C'est obligé

$\mathrm{P}$ : Finalement, oui, parce que sinon tu ne traces pas un cercle.

E : Sinon il est pas droit.

Nous pouvons lire dans cet échange une justification partielle du fait que l'on ne mentionne pas les instruments de géométrie dans les programmes de construction. En effet, l'enseignante et les élèves établissent que ces informations ne sont pas nécessaires pour réaliser la construction. Toutefois, pour que cette justification soit complète, il aurait fallu expliciter la convention selon laquelle un programme de construction ne doit comporter que les informations indispensables à la réalisation de la figure. Par ailleurs, l'enseignante formule elle-même, au moins partiellement, certains éléments discursifs de ces techniques :

$\mathrm{P}$ : Alors 2 diagonales, à partir du moment où vous utilisez le mot diagonale, ça veut dire que ça part d'un sommet et que ça va jusqu'à un autre sommet. Inutile de rajouter deux diagonales qui partent [des sommets] [...]

$\mathrm{P}$ : Vous vous adressez à des personnes qui savent tracer des figures géométriques et qui savent ce qu'est une diagonale.

L'enseignante explique ainsi qu'il est inutile de préciser la définition de diagonale puisque le lecteur la connaît. D'après la convention précédente, cette information ne doit donc pas figurer dans un programme de construction. Enfin, se référant une fois encore implicitement à cette nécessité de concision d'un programme de construction, l'enseignante justifie également l'usage de termes mathématiques :

P : On est plus dans les termes mathématiques. [...] On utilise moins d'énergie puisqu'on a moins de mots. On écrit moins de texte mais c'est plus précis.

Par conséquent, comme dans les SDA québécois, le temps praxéologique a bien avancé, sans pour autant provoquer une avancée du temps didactique.

\section{Quelles sont les conséquences pour le SDP?}

Nous avons précédemment montré (Assude $\varepsilon$ al., 2016 b.) qu'au début des SDP québécois le temps praxéologique des élèves du SDA était en avance par rapport à celui de leurs camarades (meilleure connaissance de la situation et du type de tâches demandé), puis que rapidement cette avance s'estompait et que tous les élèves de la classe paraissaient globalement en phase (les techniques proposées par les élèves du SDA étaient comparables à celles de leurs camarades). Enfin, dans deux des dispositifs québécois, nous avions pu mettre en évidence en fin de séance en classe entière, des éléments qui semblaient montrer que le temps praxéologique des élèves du SDA prenait du retard par rapport à leurs camarades.

Afin de voir ce qu'il en est pour le SDP français, regardons cet échange qui se situe au début de la séance (épisode 1 de l'annexe 3), entre Ode, l'enseignante et Nina qui, elle, n'a pas participé au SDA :

$\mathrm{O}$ : Peut-on rajouter des choses?

$\mathrm{P}$ : Oui, on peut rajouter des choses si l'on veut. Oui bien sûr Ode on peut rajouter des choses. (A la classe) Elle demande si sur la figure on peut rajouter des choses. Et je lui dis que oui on peut rajouter des choses qui peuvent nous aider. Qu'est-ce que tu entends par des choses?

$\mathrm{O}$ : Des angles droits, des codages. [...] Des sommets. [...]

$\mathrm{N}$ : Est-ce qu'on peut faire des formes géométriques?

$\mathrm{P}$ : Je ne comprends pas ce que tu veux dire.

$\mathrm{N}$ : Mais... est-ce qu'on peut faire un triangle?

$\mathrm{P}$ : Est-ce qu'on te demande de faire quelque chose? Qu'est-ce qu'on te demande de faire Nina?

Ainsi, dès le début de la séance en classe entière, alors que ses camarades découvrent à peine la situation, Ode propose d'ajouter des éléments sur la figure, notamment des sommets (c'est-à-dire très certainement le nom des sommets), technique qui était effectivement attendue par l'enseignante. A contrario, la question de sa camarade Nina, qui, ayant entendu l'enseignante préciser que l'on pouvait compléter le dessin, propose d'ajouter des figures géométriques, montre qu'elle n'a toujours pas saisi le type de tâches que l'on attendait d'elle, ce qui va dans le sens d'une certaine avance du temps praxéologique des élèves du SDA par rapport à leurs camarades. 
Karine Millon-Fauré \& al.

Par ailleurs, les productions des élèves du SDA durant l'élaboration d'un programme de construction en binôme, attestent de la mise en ouvre de techniques comparables à celles de leurs camarades, $a$ priori moins en difficulté qu'eux. Ainsi si l'on regarde la production de Léa et Lucas:

« Trace un carré et fais deux diagonales qui partent des sommets et avec ton compas, fais un cercle de centre A. »

Certes, il y a des précisions inutiles ( « diagonales qui partent des sommets » et l'évocation des outils de géométrie) et surtout des informations manquantes concernant la position relative du carré et du cercle, mais la plupart des productions de la classe comportent également ce type de maladresses. Fabio et Ode parviennent, eux aussi, à écrire un programme de construction comparable à ceux proposés par leurs camarades :

« Trace un cercle puis trace un carré à l'intérieur du cercle. Trace des diagonales à l'intérieur du carré et rajoute les codages. »

Cette fois, nous pouvons remarquer une indication concernant la position relative du carré (à l'intérieur du cercle), même si elle n'est pas encore suffisante, et l'absence d'allusions aux outils de géométrie.

Durant la deuxième séance en classe entière, l'étude des échanges atteste, pour au moins la moitié des élèves du SDA (voir partie suivante), d'un travail comparable à celui qui a pu être mené dans les autres groupes (épisode 5 de l'annexe 4):

O (elle lit le programme de construction proposé par un autre binôme) : 'Trace un carré avec une règle et une équerre'. Je le trace où je veux.

$\mathrm{F}$ : Ben non, dans le rond.

$\mathrm{O}$ : Ils le disent pas. [...] Je fais ce qu'ils disent. [...] Ils disent ça, on fait ça. 'Trace deux diagonales avec la règle'. Pourquoi à l'intérieur. Ils disent trace deux diagonales, mais ils disent pas où.

Même si cette dernière intervention est moins pertinente que les précédentes (comme elle le définit ellemême un peu plus tard, " [une diagonale va] d'un sommet à un autre, à l'intérieur »), ces échanges montrent qu'Ode a perçu le problème posé par la nécessité de préciser la position relative des figures, ce qui constitue une des principales difficultés lors de l'élaboration d'un programme de construction. Il y a même là une ébauche de technologie justifiant la nécessité de préciser ce type d'informations dans un programme de construction : sans cela, le dessinateur peut positionner les figures comme il le souhaite, même lorsqu'une position paraît plus "naturelle » que les autres.

Par ailleurs, dans un autre binôme, Arthur va être amené à soulever le problème posé par le choix d'une chronologie dans un programme de construction. Ainsi, lorsqu'il réalise que son camarade a opté pour une autre chronologie que la sienne, il cherche un moyen de mettre à l'épreuve leurs différents choix et propose d'effectuer les deux constructions, technique effectivement pertinente dans cette situation (A : « là, maîtresse, on essaye au compas pour voir c'est qui qui aura raison »). En outre, lorsqu'Arthur rencontre quelques difficultés pour effectuer le tracé demandé à partir du programme de construction qu'on lui a donné, il s'exclame « faudrait mieux faire un carré d'abord », revenant à nouveau sur le problème du choix d'une chronologie.

Pourtant, si le temps praxéologique de la plupart des élèves du SDA paraît globalement en phase avec celui de leurs camarades, on peut noter que Henry et Lucas paraissent peu à peu décrocher au cours de la seconde séance et qu'ils finissent par s'amuser ensemble sans plus se préoccuper de l'activité.

Par conséquent, il semble que, comme pour les dispositifs québécois, ce SDA a provoqué un certain avancement du temps praxéologique des élèves présents, ce qui a finalement permis de synchroniser tous les élèves pendant une bonne partie de l'activité en classe entière, même si en fin de SDP le dispositif paraît montrer pour certains élèves ses limites.

\section{FONCTION TOPOGÉNÉTIQUE}

\section{Analyse topogénétique du SDA}

Dans les SDA que nous avons observés au Québec, l'étude de la topogénèse a révélé que tous les élèves présents avaient participé au travail attendu, alors qu'ils avaient été décrits comme étant particulièrement passifs en cours. En effet, le faible effectif a permis à l'enseignante de s'assurer que chacun s'investissait bien dans le travail demandé et le fait de n'être entouré que d'élèves en difficulté a probable- 
ment donné à certains l'assurance nécessaire pour prendre la parole. Par ailleurs, les enseignantes ont choisi d'adopter durant ces dispositifs, une position topogénétique basse, notamment durant la mise en place de l'espace de questionnement précédemment décrit, ce qui a peut-être incité les élèves à prendre à leur charge une part plus grande de l'avancée des discussions.

Dans le SDA français, l'organisation du travail (espace de questionnement plus restreint et plus encadré) maintient les élèves dans une position topogénétique un peu moins haute. C'est d'ailleurs Cléa qui introduit elle-même dans le milieu plusieurs termes mathématiques que les élèves ne parviennent pas à retrouver, comme " diagonale », « codage» ou « rayon », ce qui ne s'était pas produit dans les classes québécoises. Toutefois, ce travail en petit groupe a permis à l'enseignante française de solliciter davantage ces élèves, ce qui explique peut-être leur engagement inhabituel durant toute la séance. Durant ce temps, ces élèves habituellement relativement passifs, occupent un vrai topos d'élève (ils écrivent, cherchent, sont attentifs, tentent de répondre aux questions de l'enseignant). Cette attitude paraît assez exceptionnelle puisque l'enseignante nous dit en entretien : « [l'élève qui m'a le plus surpris, c'est] Henry. Plus de confiance. Et Lucas qui était complètement inexistant, j'avais l'impression sur l'APC, d'un coup il s'est révélé. »

Analyse topogénétique de la première séance en classe entière

L'étude de la fonction topogénétique des SDA québécois (Theis \& al., 2016; Assude \& al., 2016 b.) a montré que les élèves du SDA avaient adopté, une fois de retour en classe entière, un topos plus important qu'à leur habitude et nous avons conjecturé qu'il pourrait s'agir d'un des effets de nos dispositifs. Nous allons voir s'il en est de même lors de cette expérimentation.

Au début de la première séance en classe entière, nous avons relevé (voir partie précédente) l'intervention d'Ode qui permet à l'enseignante de suggérer à tous les élèves, l'ajout d'éléments sur la figure : Ode introduit donc dans le milieu une technique pertinente pour le type de tâches proposé. Par ailleurs, durant le temps de l'élaboration en binôme d'un programme de construction, l'analyse des transcrip- tions atteste d'une réelle coopération entre Fabio et Ode, chacun prenant à sa charge une part du travail demandé. Ainsi, Fabio reprend Ode en ce qui concerne sa façon de nommer le carré (ou son orthographe) et cette dernière explique à son camarade qu'il est inutile de préciser les instruments ou le support utilisés (épisode 2 de l'annexe 3) :

F : Regarde : A, B, C, D (il identifie les sommets sur sa figure).

O : Moi j'ai marqué (elle montre sa figure).

$\mathrm{F}$ : Tu as mis deux fois le B. [...]

O : Toi tu marques [sur la feuille de papier], il sait qu'on doit prendre une feuille de papier. On va pas le faire sur le mur.

Ils échangeront ainsi jusqu'à ce qu'ils aient terminé la rédaction de leur programme de construction (environ $18 \mathrm{mn}$ ) et ils resteront investis dans la tâche pendant la totalité de la séance, occupant ainsi un véritable topos d'élève.

Il y avait également dans la classe deux groupes mixtes, composés d'un élève ayant participé au SDA et d'un élève n'y ayant pas participé, ce qui nous a permis de regarder si dans cette configuration-là, les élèves du SDA ne se laissaient pas « porter » par leurs camarades. En ce qui concerne le groupe de Medi (élève hors SDA) et d'Arthur (élève du SDA), nous pouvons voir que ce dernier tient un rôle aussi important que son camarade dans les échanges (épisode 3 de l'annexe 3). Ces deux élèves ayant choisi des chronologies différentes pour tracer la figure demandée, Arthur propose d'effectuer les deux constructions pour éprouver leurs choix, technique qui n'avait pas été évoquée dans le SDA. Il est intéressant de noter que c'est en remarquant cette pratique que l'enseignante pensera à la diffuser à la classe entière (« et c'est là que ça m'a interpellée, quand je le voyais tracer à main levée et c'est vrai que je me suis dit ça peut être utile pour tout le monde aussi [] je trouvais que c'était une trouvaille originale, je m'y attendais pas. Je pensais pas que ça allait apparaître sur cette séance » dit-elle en entretien). C'est donc Arthur qui apporte cette fois une nouvelle technique dans le milieu.

En ce qui concerne le groupe d'Henry (élève du SDA) et de Flavy (élève hors SDA), la situation est quelque peu différente. Nous constatons que Henry, qui est de très loin l'élève le plus en difficulté de la classe, reste d'abord passif durant le temps de 
Karine Millon-Fauré \& al.

recherche individuelle. Il commence finalement à écrire, efface, hésite, réécrit. Pourtant, lors du travail en binôme, il ose intervenir et convainc même sa camarade, de placer les codages sur la figure en arguant que c'est ce qu'il a fait en APC, réponse qui, même si elle ne relève pas d'une justification technologique, traduit la volonté de Henry de s'inscrire dans le travail de la classe. On notera d'ailleurs que la justification de cette technique n'avait pas non plus été donnée par l'enseignante dans le SDA, cette exigence étant plutôt présentée comme une norme à respecter.

Cette initiative d'Henry surprend l'enseignante qui lors de l'entretien post nous dit : « je pense notamment à Henry celui qu'on pensait qu'il allait se faire écraser par Flavy qui est quand même une élève brillante, il a apporté quelque chose à Flavy et j'ai vu qu'il avait tiré une satisfaction parce qu'il a mis des codages sur sa figure géométrique alors que Flavy n'y avait pas pensé et donc il a eu l'impression de vraiment lui apporter quelque chose en plus et ils ont vraiment partagé à ce moment-là ». Il est par conséquent probable que cette implication dans le travail de la part d'un élève habituellement totalement passif et le fait qu'il ait osé imposer sa pratique à une camarade a priori bien meilleure ait un lien avec le travail effectué dans le SDA. Par contre, un peu plus tard, Henry se laissera facilement convaincre par Flavy de citer les outils géométriques utilisés alors qu'il avait vu lors du SDA que ces informations étaient inutiles.

\section{Analyse topogénétique de la seconde séance}

Dans la deuxième séance en classe entière, après avoir échangé leurs programmes de construction et avoir tenté de tracer les figures décrites par leurs camarades, les binômes associés se regroupent pour réfléchir aux difficultés qu'ils ont pu rencontrer et aux manières d'améliorer leurs productions. Parmi les groupes ainsi formés, deux sont constitués de trois élèves du SDA et un élève extérieur au SDA. Dans le groupe de Henry, Lucas, Léa (tous trois ont participé au SDA) et Flavy, tous les élèves participent au début à la mise en commun (c'est même Henry qui se charge de la feuille commune). Toutefois, en milieu de séance, Lucas et Henry commencent à s'amuser et à se désintéresser de l'activité. Léa continue à s'investir dans la tâche mais c'est Flavy qui émet l'essentiel des propositions (épisode 6 de l'annexe 4).
Dans le groupe de Fabio, Ode, Arthur (tous trois ont participé au SDA) et Medi, les quatre élèves participent aux échanges pendant toute la mise en commun (épisode 7 de l'annexe 4). Ode et Fabio commencent même à critiquer le programme de construction écrit par leurs camarades. On notera au fil des échanges que leurs critiques ne se cantonnent pas au formalisme attaché à un programme de construction, mais soulèvent de véritables questions de fond :

F : Alors déjà là. Quand vous dites, trace un cercle avec ton compas. Trace un carré avec une règle et une équerre. Ton carré tu peux le tracer là, mais fallait préciser où, fallait préciser trace un carré à l'intérieur du cercle.

Cette remarque est d'autant plus inattendue que le problème posé par la position relative des figures n'a été abordé ni dans le SDA, ni dans le SDP. Ode tente ensuite de convaincre ses camarades de l'importance de préciser cette information grâce à un argument particulièrement pertinent :

$\mathrm{O}$ : Je vais inventer une figure. Tu vas me dire. Trace un rectangle et trace un cercle : tu fais quoi? [...] Tu traces un cercle au milieu de la feuille. Où tu traces un rectangle?

A : Ben avec le cercle.

$\mathrm{O}$ : Ben non. [...] moi je voulais pas que tu le traces dans le cercle je voulais que tu le traces à côté. T'as vu, t'as pas compris!

Les échanges sur la question, essentielle pour un programme de construction, de la position relative des diverses figures, durent plusieurs minutes pendant lesquelles les quatre élèves (et notamment les trois élèves du SDA) interviennent.

Ode et Fabio critiquent également les constructions de leurs camarades :

F : Ils sont où les codages?

$\mathrm{O}$ : Nulle part. Et il y a pas les diagonales.

F : Attends, attends, montre-moi ils sont où les codages?

Ils leur reprochent surtout l'approximation de leurs tracés qui fait que leur quadrilatère ne ressemble pas au carré demandé. 
F : Un carré, c'est ça un carré?

$\mathrm{O}$ : C'est un rectangle. Un carré, c'est tous les côtés de la même longueur.

Medi explique alors qu'ils n'ont pas réussi à construire un carré inscrit dans le cercle qu'ils venaient de tracer, que c'était «trop dur ». Il convient de remarquer que sa remarque est judicieuse. Le raisonnement permettant de construire un carré inscrit dans un cercle donné s'avère délicat pour des élèves de cet âge et le choix d'une autre chronologie (tracer tout d'abord le carré, puis ses diagonales et enfin le cercle ayant pour diamètre une de ces diagonales) aurait certainement facilité les constructions. Pourtant, Ode maintient sa position et tout en construisant au jugé un quadrilatère assez proche d'un carré dans le cercle qu'elle vient de tracer, elle affirme :

\section{O : Ce n'est pas dur regarde tu fais hop ! Hop ! Hop !}

Devant l'aplomb d'Ode, Medi n'ose plus rien dire. Arthur tente de refaire la construction :

A : Vous allez voir comment c'est impossible. Impossible.

Mais Ode termine toujours au jugé, le tracé en obtenant encore une fois un quadrilatère assez proche d'un carré. Medi et Arthur ne parvenant pas à se défendre, Fabio et Ode concluent très fiers d'eux :

O : Le nôtre il est tout indiqué. [...] Faites-nous une remarque, s'il y en a une.

F : Trouves-en une.

Medi intervient alors :

$\mathrm{M}$ : Trace un cercle, mais comment on va le tracer, tout petit, heu le carré.

Il veut très certainement dire que l'instruction proposée par Fabio et Ode ( « trace un carré à l'intérieur du cercle ») n'implique pas que le carré doit être inscrit dans le cercle. La position relative de ces deux figures n'est donc pas assez précise. Mais la réponse de Fabio suffit à le convaincre :

F : La maîtresse a dit qu'on ne mettait pas les mesures.
Ces épisodes illustrent l'assurance de Fabio et Ode, deux élèves en difficulté, même lorsqu'ils se trouvent en opposition avec Medi qui est pourtant un bon élève de la classe. Ils montrent comment ces deux élèves du SDA, et également dans une moindre mesure Arthur, ont su prendre ici un véritable topos d'élève, en prenant part aux échanges, en réagissant aux propositions de leurs camarades et en argumentant pour défendre leur position.

De plus, lors de la mise en commun de la deuxième séance en classe entière, Arthur intervient à plusieurs reprises, notamment pour suggérer, de façon un peu maladroite, qu'il faudrait inverser la chronologie des constructions proposées, ce qui conduira la classe à parler de l'ordre des instructions.

Cette étude nous montre que, en dépit du décrochage d'Henry et Lucas en fin de deuxième séance, les élèves du SDA ont globalement occupé un topos similaire à celui de leurs camarades durant les deux séances en classe entière.

\section{DISCUSSION ET CONCLUSION}

Nous nous étions demandé, dans cet article, quelles pouvaient être les influences du contexte sur la mise en œuvre de notre dispositif afin de réfléchir aux possibilités de diffusion de ce dernier.

Nous pouvons observer que les variations dans les contraintes institutionnelles ont effectivement provoqué des modifications dans la structure du dispositif. Ainsi le fait que Cléa se soit focalisée sur la rédaction de programmes de construction pourrait être à rapprocher des contenus des programmes français sur ce point. De même l'existence, en France, d'un dispositif préétabli susceptible de permettre un travail préparatoire avec un groupe restreint d'élèves a facilité sa mise en ouvre (ce type de pratiques est d'ailleurs institutionnellement reconnu puisque le document « Repères pour mettre en œuvre les activités pédagogiques complémentaires » écrit par le ministère de l'éducation nationale et la DGESCO propose d'utiliser ces dispositifs pour, entre autres, « anticiper certaines situations qui seront collectivement vécues dans la classe ultérieurement ${ }^{15}{ }^{5}$ ).

Mais, les spécificités de cette dernière expérimentation par rapport aux précédentes s'expliquent également par la participation des enseignantes québécoises à notre recherche collaborative. Celle-ci a en effet permis aux enseignantes, d'une part, de 
Karine Millon-Fauré \& al.

réaliser collectivement une étude approfondie des savoirs, préalable essentiel pour la conception de situations pertinentes pour le SDA et le SDP, d'autre part, de mieux saisir les enjeux du dispositif proposé et donc d'adapter leurs pratiques dans cette perspective. Leur réflexion sur la facilitation de l'engagement de l'élève dans la situation s'est progressivement enrichie, au fil des diverses phases d'analyses menées conjointement avec les chercheurs. Ensemble ils ont cherché comment laisser une place plus grande aux élèves, ce qui a conduit les enseignantes à mettre en place dans le SDA « un espace de questionnement » susceptible de favoriser l'émergence de questions et de réflexions sur les techniques à utiliser, sans pour autant compromettre l'intérêt de la séance en classe entière. L'enseignante française qui, elle, ne disposait que de l'enjeu global, a dû construire seule les moyens pour y parvenir et nous avons pu notamment constater que son guidage au cours de la séance d'aide s'avérait beaucoup plus important. De même, Cléa n'a pas senti la nécessité de légitimer ce procédé ou d'inscrire le type de tâche proposé dans le SDA par rapport à celui du SDP; alors que les enseignantes québécoises avaient débuté leur SDA en soulignant son intérêt pour les élèves choisis. Ce point nous semble important car la prise de conscience du lien entre le SDA et le SDP nous paraît susceptible de favoriser l'implication des élèves dans ce travail préparatoire et de faciliter son réinvestissement lors de la séance en classe entière. Suchaut (2013) et Toullec-Théry (2015) soulignent d'ailleurs également la nécessité pour les enseignants de renforcer le lien entre le travail effectué dans les dispositifs d'aide et dans la classe :

«C'est aux élèves qu'on laisse le soin de connecter le « système didactique auxiliaire de l'aide » au système didactique principal de la classe. Or, les élèves qui présentent des difficultés ne peuvent en avoir la responsabilité » (Toullec-Théry, 2015, p. 18).

Toutefois, même si l'enseignante française n'a pas bénéficié de l'aide apportée par la participation à une recherche collaborative, nous constatons, lors de l'étude des fonctions du dispositif de nombreuses similitudes avec les expérimentations précédentes. Nous avons ainsi pu observer que l'avancée du temps praxéologique durant le SDA avait permis de synchroniser tous les élèves de la classe pendant une bonne partie des deux séances du SDP. Il s'agit là d'une caractéristique importante de nos dispositifs par rapport aux séances de remédiation qui, comme Toullec-Théry et Marlot l'ont montré ne permettent généralement pas aux élèves de se réinsérer efficacement dans le temps didactique de la classe (ToullecThéry \& Marlot, 2012; Toullec-Théry \& Marlot, 2013; Toullec-Théry, 2015). De plus, les élèves du SDA avaient, à chaque fois, occupé un véritable topos d'élève non seulement durant le dispositif en effectif réduit, mais également durant l'essentiel du SDP. Nous conjecturons donc que ce comportement, inhabituel pour ces élèves, résulte essentiellement de la mise en place de nos expérimentations : le fait de connaître, au moins partiellement, la situation du SDP et de se retrouver en phase, voire en avance, avec le temps de la classe a pu inciter ces élèves à s'investir dans la tâche proposée. Les élèves du SDA ont ainsi réussi à réinvestir les objets de savoirs travaillés durant le dispositif d'aide, voire à élaborer de nouvelles techniques, ce qui atteste d'un engagement réel et inattendu dans l'activité proposée. C'est d'ailleurs ce changement dans l'attitude de ces élèves, habituellement peu actifs qui surprend le plus l'enseignante : «C'était ça, finalement, c'était ça l'objectif c'était entrer dans l'activité, et ils sont bien rentrés. Et je pense que tout le monde a bien participé en fait. » Ainsi, les SDA ont permis aux élèves en difficulté de retrouver, au moins temporairement un peu de valeur scolaire (Tambone, 2014), ce qui constitue une spécificité de nos dispositifs par rapport aux systèmes didactiques auxiliaires traditionnels qui travaillent essentiellement sur des objets obsolètes pour la classe :

« la valeur sociale dans la classe est liée au temps didactique. On l'acquiert en étant synchrone avec le temps didactique que l'enseignant impose à la classe » (Tambone, 2014, p. 54).

Ceci nous paraît aller dans le sens d'attester de la robustesse de notre dispositif et nous amène à envisager les possibilités de diffusion de nos dispositifs d'aide, même dans un contexte différent de celui de son élaboration (notamment sans un accompagnement comparable à celui mis en place dans une recherche collaborative, ce qui s'avère concrètement impossible à organiser pour une diffusion plus large). Cette dernière expérimentation nous a toutefois permis de prendre conscience des points qui pouvaient compromettre la reproductibilité de notre 
Karine Millon-Fauré \& al.

dispositif. Il nous semble en effet que des échanges plus approfondis avec Cléa auraient pu permettre d'enrichir cette mise en ouvre en l'amenant à moins se focaliser sur l'aspect formel des programmes de construction, à réduire son guidage du travail des élèves et à renforcer le lien avec le travail effectué en classe entière. Il faut reconnaître que l'appropriation de ce dispositif nécessite un changement délicat dans les pratiques des enseignants qui ne conçoivent généralement l'aide apportée aux élèves que sous forme de remédiation. Nous pensons donc qu'un certain accompagnement - selon des modalités qui restent à définir - s'avère indispensable pour initier ce changement. Sans vouloir imposer le modèle élaboré lors de notre recherche collaborative, nous pensons qu'une réflexion préalable des enseignants sur les moyens qui renforceraient les liens entre le SDA et le SDP et faciliteraient l'entrée des élèves dans le milieu, conforterait les fonctions de notre dispositif. De même, nous conjecturons que le fait d'envisager, pour le SDA, d'autres formes d'enseignement qui encourageraient les interactions sans chercher à apporter des réponses exactes et définitives, permettrait aux élèves de confronter leurs techniques avec celles de leurs camarades et de s'acheminer vers des praxéologies plus pertinentes. Il ne s'agit toutefois que de réflexions résultant d'une première expérimentation avec un enseignant n'ayant pas participé à notre recherche collaborative et il convient à présent d'étudier d'autres mises en œuvre de notre dispositif dans des contextes différents, afin de s'assurer de la pérennité de ses fonctions et d'affiner nos modalités de diffusion.

\section{NOTES}

1. On appelle « objets sensibles », les objets de savoir «que l'enseignant professe et sur lesquels porte l'action enseignante visible ». On qualifie d'objets non sensibles, les objets qui ne sont pas sensibles qu'il s'agisse « d'objets "désensibilisés" (professés il y a quelque temps) ou "insensibles » (non enseignés mais pertinents pour l'étude des savoirs sensibles) »(Mercier, 1996, p. 348).

2. Extrait de « Apprentissages géométriques et résolution de problèmes » Hatier Ermel Cycle 3 p. 437.

3. Organisation du temps scolaire dans le premier degré et des activités pédagogiques complémentaires ; B.O n 6 du 7 février 2013 ; p.6.
4. Programme de l'école primaire. B.O No 3 du 19 juin 2008 Hors-série.

5. Le cycle 3 correspond aux classes de CE2, CM1 et CM2 (élèves de 8 à 10 ans).

6. École Documents d'application des programmes mathématiques cycle des approfondissements (cycle 3) ; ministère de la Jeunesse, de l'Éducation nationale et de la Recherche, 2002.

7. Depuis la rentrée 2016, le cycle 3 correspond aux classes de CM1, CM2 et 6 $6^{\text {e }}$ (élèves de 9 à 11 ans).

8. Programme d'enseignement du cycle 3 ; B.O spécial no 11 du 26 novembre 2015 .

9. Programme d'enseignement du cycle 3 ; B.O spécial no 11 du 26 novembre 2015.

10. MELS (2009). Programme de Formation de l'École Québécoise. Progression des apprentissages. Gouvernement du Québec; p. 15.

11. Pour plus de précisions, voir Assude $\&$ al., 2016 a. 12. On parle de rapport d'idonéité lorsqu'un élève entretient avec un objet de savoir un rapport personnel en adéquation avec les attentes institutionnelles (Mercier, 1999).

13. On désigne par rétroactions les réponses du milieu aux actions des élèves.

14. Durant l'institutionnalisation du SDP, l'enseignante ajoutera trois autres contraintes que les élèves devront suivre pour élaborer un programme de construction : respecter un ordre, ne pas tenir compte de l'orientation de la figure, utiliser des verbes au présent de l'impératif.

15. Repères pour mettre en œuvre « les activités pédagogiques complémentaires ». Octobre 2013 MEN-DGESCO.

\section{RÉFÉRENCES}

Assude, T. (2005). Time management in the work economy of a class, a case study : integration of CABRI in primary school mathematics teaching. Educational Studies in Mathematics, 59, 183-203.

Assude, T., Koudogbo, J., Millon-Fauré, K., Morin, M.-P., Tambone, J., Theis, L. (2016 a). Mise à l'épreuve des fonctions d'un dispositif d'aide aux élèves en difficulté en mathématiques. Canadian Journal of Science, Mathematics and Technology Education, 16(1), 1-35.

Assude, T., Millon-Faure, K., Koudogbo, J., Morin, M.-P., Tambone, J. et Theis, L. (2016 b). Du rapport entre temps didactique et temps praxéologique dans des dispositifs d'aide associés à une classe. Recherches en didactique des mathématiques, 36(2), 197-226.

Bednarz, N. (dir) (2013). Recherche collaborative et pratique enseignante. Regarder ensemble autrement. LHarmattan : Paris. 
Karine Millon-Fauré \& al.

Brousseau, G. \& Centeno, J. (1991). Rôle de la mémoire didactique de l'enseignant. Recherche en didactique des mathématiques, 11(2), 167-210.

Chevallard, Y. (1995). La fonction professorale : esquisse d'un modèle didactique. Dans R. Noirfalis \& M.-J. Perrin-Glorian (dir.), Actes de la VIII école d'été de didactique des mathématiques (p. 83-122). ClermontFerrand : IREM.

Chevallard, Y. (1999). Lanalyse des pratiques en théories anthropologique du didactique. Recherches en didactiques des mathématiques, 19(2), 221-266.

Chevallard, Y. (2010). Le sujet apprenant entre espace et dispositif. Commentaires depuis la théorie anthropologique du didactique. Communication présentée aux journées du Lisec Gérardmer, [en ligne : http://yves. chevallard.free.fr/spip/spip/IMG/pdf/Commentaires_ depuis_la_TAD_YC_.pdf].

Desgagné, S. (1997). Le concept de recherche collaborative : l'idée d'un rapprochement entre chercheurs universitaires et praticiens enseignants. Revue des sciences de l'éducation, 31(2), 245-258.

Desgagné, S. \& Bednarz, N. (2005). Médiation entre recherche et pratique en éducation : faire de la recherche " avec » plutôt que "sur » les praticiens. Revue des sciences de l'éducation, 23(2), 371-393.

Giroux, J. (2014). Les difficultés d'enseignement et d'apprentissage des mathématiques : historique et perspectives théoriques. Dans C. Mary, L. Deblois, H. Squalli \& L. Theis (dir.), Recherches sur les difficultés d'enseignement et d'apprentissage des mathématiques (p. 11-44). Québec : Presses Universitaires du Québec.

Lahanier-Reuter, D. (1999). Éléments d'analyse de descriptions en mathématiques. Petit x, 53, 27-46.

Leutenegger, F. (2009). Le temps d'instruire. Approche clinique et expérimentale du didactique ordinaire en mathématiques. Berne: Peter Lang.

Mary, L., Deblois, L., Squalli, H. \& Theis, L. (dir.) (2014). Recherches sur les difficultés d'enseignement et d'apprentissage des mathématiques. Québec : Presses Universitaires du Québec.

Mercier, A. (1996). La création d'ignorance, condition de l'apprentissage. Revue des sciences de l'éducation, 22(2), 345-363.

Mercier, A. (1999). L'espace temps didactique, études du didactique en sciences de l'éducation. Note pour l'habilitation à diriger des recherches, université d'AixMarseille 1, Marseille.

Millon-Fauré, K. (2011). Les répercussions des difficultés langagières des élèves sur l'activité mathématique en classe : le cas des élèves migrants. Thèse de doctorat, université d'Aix-Marseille 1, Marseille.

Schubauer-Leoni, M-L., Leutenegger, F, Ligozat, F., Fluckiger, A. (2007). Un modèle de l'action conjointe professeur-élèves : les phénomènes didactiques qu'il peut/doit traiter. Dans G. Sensevy \& A. Mercier (dir.), Agir ensemble. Éléments de théorisation de l'action conjointe du professeur et des élèves (p. 51-91). Rennes : Presses Universitaires de Rennes.
Sensevy, G., Mercier, A. \& Schubauer-Leoni, M.-L. (2000). Vers un modèle de l'action didactique du professeur. À propos de la course à 20. Recherches en didactique des mathématiques, 20(3), 263-304.

Suchaut, B. (2013). Plus de maîtres que de classes, analyse des conditions de l'efficacité du dispositif (Rapport de recherche), [en ligne: http://www.cafepedagogique. net/lexpresso/Documents/suchaut180313.pdf].

Tambone, J., Mercier, A. (2003). Larticulation entre classe et groupe d'adaptation de l'aide à dominante pédagogique, en France, pose questions sur la notion de système didactique. Dans G. Chatelanat, \& G. Pelgrims, (dir.), Éducation et enseignement spécialisés : ruptures et intégrations (p. 195-213). Paris, Bruxelles : Raisons Éducatives, De Boeck Université.

Tambone, J. (2014). Enseigner dans un dispositif auxiliaire : le cas du regroupement d'adaptation et de sa relation avec la classe d'origine de l'élève. Les Sciences de l'éducation - Pour l'Ère nouvelle, 47(2), 51-71.

Theis, L., Assude, T., Tambone, J., Morin, M.-P., Koudogbo, J. et Marchand, P. (2014). Quelles fonctions potentielles d'un dispositif d'aide pour soutenir la résolution d'une situation-problème mathématique chez des élèves en difficulté du primaire? Éducation et francophonie, 42(2), 160-174.

Theis, L., Morin, M.-P., Tambone J., Assude T., Koudogbo J., et Millon-Fauré, K. (2016). Quelles fonctions de deux systèmes didactiques auxiliaires destinés à des élèves en difficulté lors de la résolution d'une situation-problème mathématique? Annales de didactique et de sciences cognitives. Revue Internationale de Didactique des Mathématiques, 21,9-38.

Toullec-Théry, M. \& Marlot, C. (2012). Efficacité et déterminations des pratiques d'Aide Personnalisée à l'école primaire : une approche didactique. Recherches en éducation, Hors-série 4, 81-97.

Toullec-Théry, M. \& Marlot, C. (2013). Les déterminations $\mathrm{du}$ phénomène de différenciation didactique passive dans les pratiques d'aide ordinaire à l'école élémentaire. Revue française de pédagogie, 182, 41-54.

Toullec-Théry, M. (2015). Des politiques françaises en matière d'éducation centrées sur l'individualisation, la personnalisation plus que sur le collectif : quels effets sur les apprentissages des élèves? Une contribution didactique. Conseil national d'évaluation $d u$ système scolaire. [http://www.cnesco.fr/wp-content/ uploads/2016/09/toullecl.pdf]. 


\section{ANNEXES}

ANNEXE 1 : ACTIVITÉS PROPOSÉES DANS LE SDA LORS DE LA RECHERCHE COLLABORATIVE AU QUÉBEC

Tu dois décrire la figure géométrique suivante afin que ton partenaire puisse la reproduire avec précision.

Attention ! Il ne doit pas la voir !

Terme à ne pas prononcer : carré

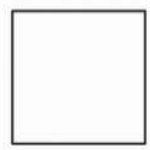

Mathématique - Géométrie

Tu dois décrire la figure géométrique suivante afin que ton partenaire puisse la reproduire avec précision.

Attention! II ne doit pas la voir!

Terme à ne pas prononcer : triangle

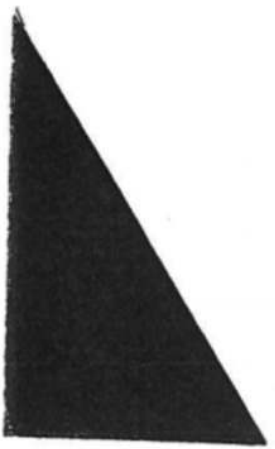


Karine Millon-Fauré \& al.

\section{ANNEXE 2 : TABLEAU SYNOPTIQUE DE LA SÉANCE DU SDA}

Dans les trois tableaux synoptiques, les prénoms des six élèves du SDA sont notés en gras. Les références à un épisode analysé dans l'article sont indiquées en italique.

Participants : P (professeur) et Arthur; Ode; Lucas; Fabio; Léa; Henry

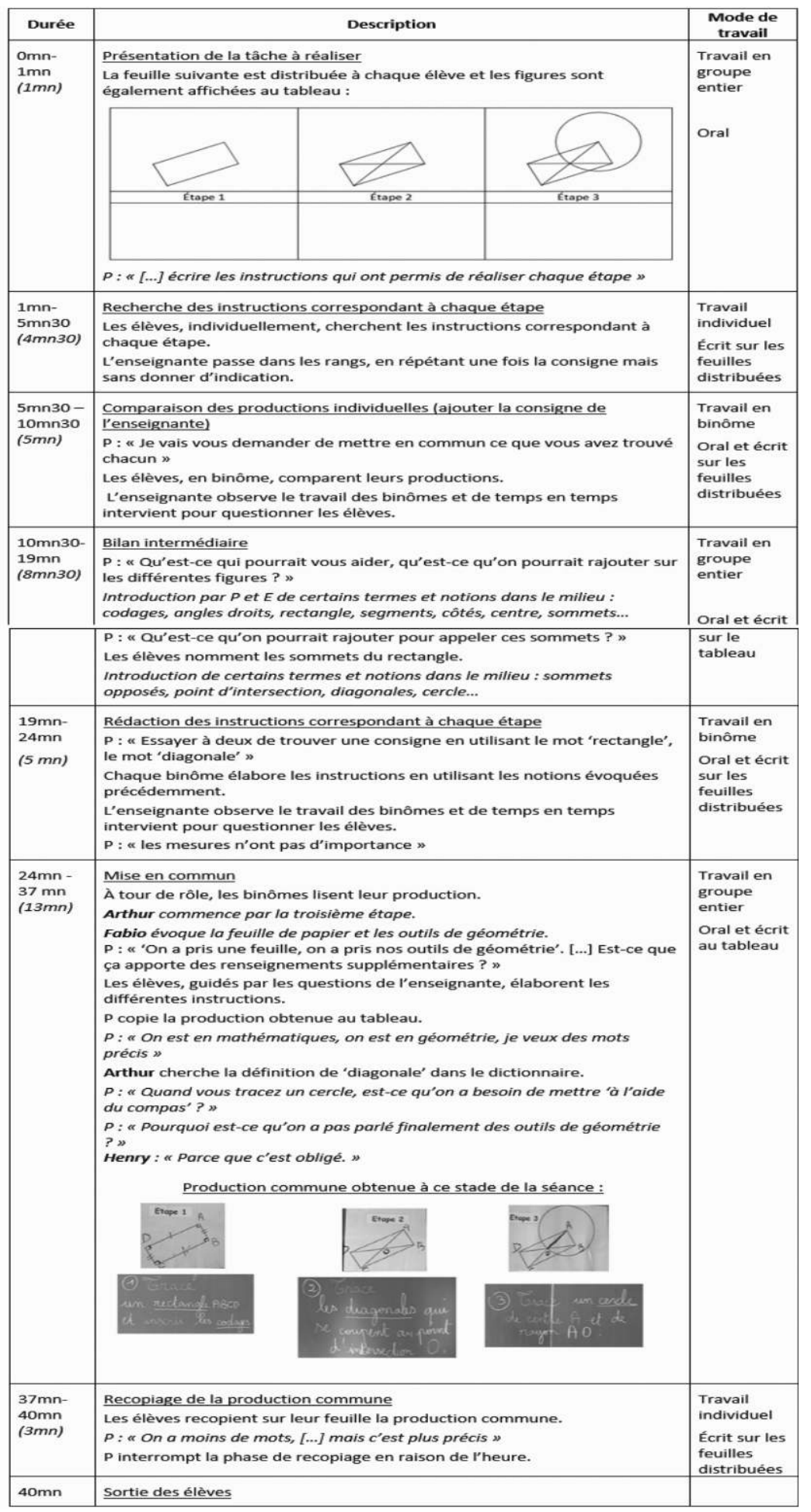


Karine Millon-Fauré \& al.

\section{ANNEXE 3 : TABLEAU SYNOPTIQUE DE LA PREMIÈRE SÉANCE DU SDP}

Dans les trois tableaux synoptiques, les prénoms des six élèves du SDA sont notés en gras. Les références à un épisode analysé dans l'article sont indiquées en italique.

Participants : P et la classe entière (dont les élèves du SDA : Arthur; Ode; Lucas; Fabio; Léa; Henry)

\begin{tabular}{|c|c|c|}
\hline Durée & Description & $\begin{array}{c}\text { Mode de } \\
\text { travail }\end{array}$ \\
\hline $\begin{array}{l}\text { Omn- } \\
3 \mathrm{mn} 30 \\
(3 \mathrm{mn} 30)\end{array}$ & $\begin{array}{l}\text { Présentation de l'activité } \\
\text { Distribution d'une figure parmi les cinq suivantes à chaque élève : } \\
\mathrm{P}: \text { : vous allez rédiger des instructions qui vont permettre à quelqu'un qui } \\
\text { ne voit pas cette figure de la refaire. " } \\
\text { Lucas : " c'est ce qu'on a fait en } A P C \text {." }\end{array}$ & $\begin{array}{l}\begin{array}{l}\text { Classe } \\
\text { entière }\end{array} \\
\text { Oral }\end{array}$ \\
\hline $\begin{array}{l}3 \mathrm{mn} 30- \\
12 \mathrm{mn} 40 \\
(9 m n 10)\end{array}$ & $\begin{array}{l}\text { Rédaction des instructions de construction (1) } \\
\text { Les élèves essaient individuellement d'écrire les instructions de construction } \\
\text { correspondant à leur figure. } \\
\text { P passe dans les rangs en observant le travail effectué, répond à quelques } \\
\text { questions concernant la compréhension de la consigne. } \\
\text { Épisode } 1 \text { : échanges entre Ode et l'enseignante, puis Nina et l'enseignante. } \\
\text { Ode demande si l'on peut ajouter des choses sur la figure. P confirme. Nina } \\
\text { demande si l'on peut ajouter des formes géométriques. }\end{array}$ & $\begin{array}{l}\text { Travail } \\
\text { individuel } \\
\text { Écrit sur } \\
\text { une feuille }\end{array}$ \\
\hline $\begin{array}{l}12 \mathrm{mn} 40- \\
37 \mathrm{mn} 40 \\
(25 \mathrm{mn})\end{array}$ & $\begin{array}{l}\text { Rédaction des instructions de construction (2) } \\
P \text { : « Vous allez essayer de mettre en commun tout ce que vous avez trouvé » } \\
\text { Les élèves essaient en binôme de rédiger les instructions de construction } \\
\text { correspondant à leur figure en s'appuyant sur leur recherche individuelle (les } \\
\text { deux élèves du binôme ont eu la même figure). } \\
\text { Épisode } 2 \text { : Binôme Fabio/Ode. Implication des deux élèves (nombreux } \\
\text { échanges); réflexions sur la nécessité de préciser les outils de construction, } \\
\text { la façon de nommer un carré, la définition de 'diagonale' ... } \\
\text { Épisode } 3 \text { : Binôme Medi/Arthur. Implication des deux élèves (nombreux } \\
\text { échanges); réflexions sur le choix de la chronologie pour la construction; } \\
\text { Arthur propose de construire les figures afin de tester leurs programmes de } \\
\text { construction; P expose la technique à la classe } \\
\text { Épisode } 4 \text { : Binôme Elavy/Henry. Travail important de Elavy, Intervention } \\
\text { d'Henry concernant les codages. } \\
\text { Dans le binôme Lucas/Léa : implication des deux élèves. } \\
\text { P passe dans les rangs en observant le travail effectué, demande à certains } \\
\text { élèves d'expliciter leur travail. }\end{array}$ & $\begin{array}{l}\text { Travail en } \\
\text { binôme } \\
\text { Oral et } \\
\text { écrit sur } \\
\text { une feuille } \\
\text { commune }\end{array}$ \\
\hline $\begin{array}{l}37 \mathrm{mn} 30- \\
45 \mathrm{mn}\end{array}$ & Bilan & $\begin{array}{l}\text { Classe } \\
\text { entière }\end{array}$ \\
\hline (7mn30) & $\begin{array}{l}\text { P répond à quelques questions des élèves et explique ce qui sera fait par la } \\
\text { suite concernant cette activité (notamment lors de la séance suivante). } \\
\text { Ramassage des feuilles } \\
\text { P : " Quelle matière vous avez eu l'impression de faire, aujourd'hui ? [...] Que } \\
\text { géométrie? [...] Effectivement on a fait de l'expression écrite, on a fait de la } \\
\text { géométrie [...] et on a fait du vocabulaire.» }\end{array}$ & Oral \\
\hline
\end{tabular}


Karine Millon-Fauré \& al.

\section{ANNEXE 4 : TABLEAU SYNOPTIQUE DE LA DEUXIÈME SÉANCE DU SDP}

Dans les trois tableaux synoptiques, les prénoms des six élèves du SDA sont notés en gras. Les références à un épisode analysé dans l'article sont indiquées en italique.

Participants : P et la classe entière (dont les élèves du SDA : Arthur; Ode; Lucas; Fabio; Léa; Henry)

\begin{tabular}{|c|c|c|}
\hline Durée & Description & $\begin{array}{c}\text { Mode de } \\
\text { travail }\end{array}$ \\
\hline $\begin{array}{l}0 m n- \\
6 m n 20 \\
(6 m n 20)\end{array}$ & $\begin{array}{l}\text { Présentation de l'activité } \\
\text { P: « sur quoi on avait travaillé ? » } \\
\text { P présente l'activité du jour } \\
\text { P: « je vais distribuer à certains groupes le travail qu'ont fait un autre } \\
\text { groupe et on va voir si vos camarades arrivent [...] à la même figure que } \\
\text { vous. » } \\
\text { Mise en binôme (les mêmes binômes que la dernière fois) } \\
\text { Distribution des productions de la dernière fois (l’orthographe ayant été } \\
\text { rectifiée par l'enseignante) et d’une feuille blanche par binôme. }\end{array}$ & $\begin{array}{l}\text { Classe entière } \\
\text { Oral }\end{array}$ \\
\hline $\begin{array}{l}6 \mathrm{mn} 20- \\
13 \mathrm{mn} \\
(6 \mathrm{mn} 40)\end{array}$ & $\begin{array}{l}\text { Construction des figures à partir du programme de construction } \\
\text { Les élèves, en binôme, construisent la figure correspondant aux } \\
\text { instructions qu'ils ont reçues. } \\
\text { Épisode } 5 \text { : Binôme Ode-Fabio. Les élèves construisent à tour de rôle la } \\
\text { figure; réflexion sur la position relative des figures élémentaires } \\
\text { constituant la figure à tracer. } \\
\text { Binôme Arthur-Medi : les deux élèves tentent l'un après l'autre de } \\
\text { construire la figure correspondant aux instructions. Ils rencontrent des } \\
\text { difficultés pour tracer le carré inscrit dans le cercle. Arthur propose de } \\
\text { modifier la chronologie proposée. } \\
\text { Binôme Henry-Flavy: Flavy trace la figure. Henry n'intervient quasiment } \\
\text { pas. }\end{array}$ & $\begin{array}{l}\text { Travail en } \\
\text { binồme } \\
\text { Oral et écrit } \\
\text { sur la feuille } \\
\text { commune }\end{array}$ \\
\hline $\begin{array}{l}13 \mathrm{mn}- \\
15 \mathrm{mn} 40 \\
(2 \mathrm{mn} 40)\end{array}$ & $\begin{array}{l}\text { Comparaison de la figure construite avec celle de départ } \\
\text { Les élèves vont chercher la figure correspondant au programme de } \\
\text { construction qưils ont reçu et ils la comparent à leur tracé. } \\
\text { Binôme Fabio-Ode : ils constatent des différences. Ode : « ils ont mal } \\
\text { précisé n }\end{array}$ & $\begin{array}{l}\text { Travail en } \\
\text { binôme } \\
\text { Oral }\end{array}$ \\
\hline $\begin{array}{l}15 \mathrm{mn} 40- \\
20 \mathrm{mn} 20 \\
(4 \mathrm{mn} 40)\end{array}$ & $\begin{array}{l}\text { Bilan intermédiaire } \\
\text { P: « Quand on dit 'vérifier', ça veut dire quoi ? » } \\
\text { Réflexions sur les techniques à mettre en œuvre pour comparer les } \\
\text { figures } \\
\text { P: « pour voir ce qui va pas [...] et peut-être trouver une amélioration au } \\
\text { texte, je vais regrouper les binômes qui ont écrit et les binômes qui ont } \\
\text { réalisé cette figure » } \\
\text { Mise en groupe de quatre élèves : chaque groupe est constitué des deux } \\
\text { binômes qui ont échangé leur programme de construction. }\end{array}$ & $\begin{array}{l}\text { Classe entière } \\
\text { Oral }\end{array}$ \\
\hline $\begin{array}{l}20 \mathrm{mn} 20- \\
40 \mathrm{mn} \\
(19 \mathrm{mn} 40)\end{array}$ & $\begin{array}{l}\text { Remise en question des programmes de construction proposés (1) } \\
\text { Les quatre élèves réfléchissent aux causes des écarts entre la figure } \\
\text { tracée et celle de départ. Ils essaient d'améliorer les programmes de } \\
\text { construction. } \\
\text { P passe de groupe en groupe et par ses questions, aide les élèves à } \\
\text { améliorer leurs programmes de construction. } \\
\text { Épisode } 6 \text { : Groupe Léa-Lucas/Henry-Flavy: implication des quatre } \\
\text { élèves au départ; discussion avec l'enseignante, qui les guide pour } \\
\text { rectifier le lexique choisi;; Flavy et Léa terminent le travail alors que } \\
\text { Henry et Lucas s'amusent. } \\
\text { Épisode 7: Groupe Fabio-Ode/Arthur-Medi : Implication des quatre } \\
\text { élèves pendant toute la phase. Fabio et Ode critiquent le programme de } \\
\text { construction d'Arthur et Medi (non précision des positions relatives des } \\
\text { figures élémentaires); Fabio et Ode critiquent la figure tracée par } \\
\text { Arthur et Medi (il manque les codages, les diagonales; le quadrilatère } \\
\text { n'est pas un carré); Arthur et Medi tentent de se défendre. Ils affirrment } \\
\text { également que la figure est impossible à construire à partir du } \\
\text { programme proposé. Échanges avec P concernant ce point et le } \\
\text { problème posé par le choix de la chronologie. }\end{array}$ & 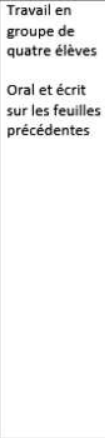 \\
\hline $\begin{array}{l}40 m n- \\
49 m n 50 \\
(9 m n 50)\end{array}$ & $\begin{array}{l}\text { Bilan } \\
\text { P: « qu'est-ce que vous auriez aimé avoir en plus ou qu'est-ce qui aurait } \\
\text { dû être modifié ? " } \\
\text { Arthur propose de faire le carré avant le cercle. } \\
\text { P guide la réflexion des élèves par ses questions et note au fur et à } \\
\text { mesure au tableau les points à retenir. } \\
\text { Points inscrits } \\
\text { au tableau à } \\
\text { la fin de cette } \\
\text { phase : }\end{array}$ & $\begin{array}{l}\text { Travail en } \\
\text { classe entière } \\
\text { Oral et écrit } \\
\text { au tableau }\end{array}$ \\
\hline $\begin{array}{l}49 \mathrm{mn} 50- \\
58 \mathrm{mn} 50 \\
(9 \mathrm{mn})\end{array}$ & $\begin{array}{l}\text { Remise en question des programmes de construction proposés (2) } \\
\mathrm{P}: \text { \& vous avez } 5 \mathrm{mn} \text { pour reprendre tout ce que vous venez de voir, tout } \\
\text { ce que vous venez de dire. ») } \\
\text { Les quatre élèves essaient d'améliorer leurs programmes de construction } \\
\text { en se servant des points évoqués lors du bilan. } \\
\text { P passe de groupe en groupe et par ses questions, aide les élèves à } \\
\text { améliorer leurs programmes de construction, en se référant notamment } \\
\text { aux points notés au tableau. } \\
\text { Groupe Fabio-Ode/Arthur-Medi : Implication des quatre élèves. Échanges } \\
\text { avec l'enseignante (sur le choix de la chronologie...)) } \\
\text { Groupe Léa-Lucas/Henry-Flavy : Flavy et Léa travaillent; Henry et Lucas } \\
\text { s'amusent. }\end{array}$ & $\begin{array}{l}\begin{array}{l}\text { Travail en } \\
\text { groupe de } \\
\text { quatre élèves }\end{array} \\
\text { Oral et écrit } \\
\text { sur les feuilles } \\
\text { précédentes }\end{array}$ \\
\hline $\begin{array}{l}58 \mathrm{mn} 30- \\
1 \mathrm{hO4mn} \\
(5 \mathrm{mn} 30)\end{array}$ & $\begin{array}{l}\text { Clôture de l'activité. } \\
\text { Prappelle les règles de travail en groupe. } \\
\text { Prappelle tous les points qui ont été notés au tableau. }\end{array}$ & $\begin{array}{l}\text { Travail en } \\
\text { classe entière } \\
\text { Oral }\end{array}$ \\
\hline
\end{tabular}

